\title{
Deposición electroforética de una porcelana dental sobre acero inoxidable austenítico 304
}

\author{
G. VARGAS, A. MUÑOZ, J. MÉNDEZ, M. MÉNDEZ, P. MONDRAGÓN \\ CINVESTAV - IPN, Unidad Saltillo, Saltillo Coahuila. México.
}

\begin{abstract}
Una técnica alternativa para la fabricación de restauraciones dentales con porcelana, es la deposición electroforética (EPD). En este trabajo depósitos de porcelana dental sobre acero inoxidable fueron obtenidos por EPD utilizando como medios de suspensión: agua, etanol e isopropanol. Se estudió el efecto de la concentración de porcelana, de la intensidad del campo eléctrico y del medio de suspensión sobre la cantidad de masa depositada. Todas las pruebas fueron realizadas a voltaje constante, utilizando un tiempo de deposición de $3 \mathrm{~min}$. La porcelana dental utilizada presentó un intervalo de tamaño de partícula entre 1,5 y 2,3 $\mu \mathrm{m}$. Los resultados mostraron que la porcelana en el medio acuoso presentó la mayor velocidad de deposición, en comparación con el etanol y el isopropanol. Sin embargo, debido a problemas relacionados con la baja estabilidad de las suspensiones y a la hidrólisis del agua, los mejores acabados superficiales de los depósitos se obtuvieron utilizando etanol. Estos resultados fueron obtenidos aplicando voltajes entre 70 y $150 \mathrm{~V}$, para un tiempo de deposición de 3 min. El uso de isopropanol generó depósitos heterogéneos debido a problemas relacionados con la baja estabilidad de las suspensiones y con reacciones electroquímicas en el electrodo de trabajo, derivadas de la aplicación de voltajes altos.
\end{abstract}

Palabras clave: Deposición electroforética (EPD), porcelana, acero inoxidable, suspensiones.

\section{Electrophoretic deposition of dental porcelain on stainless steel 304}

An alternative technique for the production of dental restorations with porcelain, is the electrophoretic deposition (EPD). In this work, deposits of dental porcelain on stainless steel were obtained by EPD using water, ethanol and isopropanol as suspension mediums. The effect of the porcelain concentration, the intensity of the electric field and the suspension medium on the quantity of deposited mass were studied. All the tests were carried out at constant voltage, using a deposition time of $3 \mathrm{~min}$. The particle size of the dental porcelain was in the range between 1.5 and $2.3 \mu \mathrm{m}$ in the suspension. The results showed that the porcelain in the aqueous medium presented the major deposition velocity, in comparison with both ethanol and isopropanol. However, due to low the low suspension stability and hydrolysis of the water problems, the best coating finishes were obtained with ethanol. These results were obtained applying voltages between 70 and $150 \mathrm{~V}$, for a deposition time of $3 \mathrm{~min}$. Using isopropanol heterogeneous deposits were obtained due to the low suspension stability and to electrochemical reactions problems in the working electrodes, resulted from the high voltages application.

Key words: Electrophoretic deposition (EPD), porcelain, stainless steel, suspensions.

\section{INTRODUCCIÓN}

Las porcelanas dentales están constituidas generalmente por feldespato de potasio $\left(\mathrm{K}_{2} \mathrm{O}, \mathrm{Al}_{2} \mathrm{O}_{3}, 6 \mathrm{SiO}_{2}\right)$, cuarzo $\left(\mathrm{SiO}_{2}\right)$, caolín y un pequeño porcentaje de óxidos metálicos que actúan como elementos opacificadores y de pigmentación que permiten igualar la apariencia natural en las restauraciones dentales. Algunas de las porcelanas más recientes contienen cantidades importantes de $\mathrm{Na}_{2} \mathrm{O}$, las cuales permiten obtener porcelanas de bajo punto de fusión (1). Las fases presentes en las porcelanas están determinadas por las proporciones en que se encuentran los componentes y por el tratamiento a que son sometidas. El contenido de leucita $\left(\mathrm{KAlSi}_{2} \mathrm{O}_{6}\right)$, permite elevar el coeficiente de expansión térmica de las porcelanas a valores similares a los de las aleaciones metálicas de uso dental $(1,2)$.

El esmaltado es la técnica de uso más frecuente para aplicar la porcelana dental. Un método alterno, de fácil operación, bajo costo y que permite recubrimientos densos y uniformes es la deposición electroforética (EPD). En la EPD intervienen dos procesos: la electroforesis y la deposición.
La electroforesis es un fenómeno asociado al movimiento de partículas, que tiene lugar al aplicar un campo eléctrico a una suspensión coloidal estable. Este fenómeno está directamente relacionado con las propiedades electroquímicas superficiales que adquieren las partículas cuando se hallan inmersas en un medio líquido. Para asegurar un flujo uniforme de partículas depositables se debe de tener en cuenta (3):

-Que la suspensión sea suficientemente estable, dotando a las partículas de una elevada movilidad electrocinética, y

-Que el campo eléctrico creado en la suspensión sea homogéneo, en función de la geometría del sistema, y lo suficientemente elevado para hacer que las partículas se muevan en la dirección deseada.

El segundo proceso que tiene lugar durante el proceso es la deposición de las partículas sobre el electrodo de trabajo formando un cuerpo en verde relativamente compacto $(3,4)$.

La estabilidad de una suspensión coloidal es un factor clave en el procesamiento de materiales por EPD, ya que de 
ésta depende en gran medida, la reproducibilidad y la optimización de los parámetros de procesamiento (5). Los medios de suspensión acuosos son preferibles, por su bajo costo, en el procesamiento por EPD de materiales cerámicos. Sin embargo, se pueden presentar dificultades asociadas a la descomposición electroquímica del agua en los electrodos (electrolisis). Fenómeno que ocurre a potenciales relativamente bajos $(\sim 5$ V). Dependiendo de la polaridad del electrodo pueden ocurrir las siguientes reacciones:

$$
\begin{aligned}
& 2 \mathrm{H}_{2} \mathrm{O}+2 \mathrm{e}^{-} \rightarrow \uparrow \mathrm{H}_{2}+2 \mathrm{OH}^{-} \\
& 4 \mathrm{OH}^{-}+2 \mathrm{H}_{2} \mathrm{O} \rightarrow \uparrow \mathrm{O}_{2}+4 \mathrm{e}^{-}
\end{aligned}
$$

La producción de $\mathrm{H}_{2}$ en el cátodo y de $\mathrm{O}_{2}$ en el ánodo provoca burbujeo en los electrodos, lo cual puede dañar el depósito de forma irreversible $(3,4)$.

Otros medios de suspensión utilizados son los solventes orgánicos. Estos medios presentan una constante dieléctrica baja disminuyendo el efecto de la electrólisis, por lo que se pueden obtener recubrimientos más homogéneos que en medios acuosos. Sin embargo, debido a su elevada resistividad, es necesario aplicar potenciales eléctricos relativamente altos, que pueden sobrepasar el potencial de casi cualquier metal, generando también problemas en los electrodos $(3,4,6)$. Este problema consiste en la degradación del electrodo por efecto electroquímico; provocando el desprendimiento de iones metálicos, y por lo tanto, la posible contaminación del depósito o bien de la interfase depósito-substrato $(6,7)$. Además de interferir con el proceso de deposición reduciendo la eficiencia de la corriente. Dentro de este tipo de medios los más utilizados son el etanol y el isopropanol.

En relación al recubrimiento de porcelanas dentales por EPD existen dos patentes las cuales se describen brevemente a continuación. En 1981, H. W. Hennicke (8) patentó un aparato de EPD para depositar capas intermedias de zinc, entre el substrato metálico y la porcelana dental en la fabricación de puentes y coronas dentales. Para llevar a cabo la EPD utilizaron una mezcla de un compuesto orgánico no especificado y bromuro de potasio a una concentración de $1.5 \mathrm{~g} / 1$. En 1984 los alemanes F. K. Gutersloh y W. Harsewinkel (9), patentaron un nuevo método para la fabricación de puentes y coronas dentales por EPD. Este método consistió básicamente en la utilización de dos celdas de deposición y una fuente de poder con la cual se controlan las condiciones eléctricas del proceso. Las suspensiones formuladas de acuerdo a esta patente, se preparan con $100 \mathrm{~g}$ de porcelana, $50 \mathrm{ml}$ de agua destilada y $50 \mathrm{ml}$ de metil-celulosa. Los voltajes para llevar a cabo la aplicación de las porcelanas fueron de 4 a $40 \mathrm{~V}$.

El objetivo principal del presente trabajo fue el estudio del efecto de la concentración de porcelana, de la intensidad del campo eléctrico y del medio de suspensión sobre la cantidad de masa depositada y analizar el acabado superficial de los depósitos.

\section{PARTE EXPERIMENTAL}

2.1 Identificación y acondicionamiento de las materias primas.

Porcelana dental. Para realizar las pruebas de EPD, se utilizó una porcelana disponible comercialmente (Body Ceramco A1). Como se puede apreciar en la Tabla 1 los constituyentes principales son $\mathrm{Al}_{2} \mathrm{O}_{3}, \mathrm{SiO}_{2}, \mathrm{~K}_{2} \mathrm{O}$ (constituyentes de la
TABLA 1. COMPOSICIÓN QUÍMICA DE LA PORCELANA UTILIZADA

\begin{tabular}{|c|c|}
\hline Compuesto & \% peso \\
\hline $\mathrm{SiO}_{2}$ & 58,28 \\
\hline $\mathrm{Al}_{2} \mathrm{O}_{3}$ & 21,82 \\
\hline $\mathrm{K}_{2} \mathrm{O}$ & 8,75 \\
\hline $\mathrm{Na}_{2} \mathrm{O}$ & 6,05 \\
\hline $\mathrm{CaO}$ & 1,96 \\
\hline $\mathrm{MgO}$ & 1,74 \\
\hline $\mathrm{SnO}$ & 0,47 \\
\hline $\mathrm{Fe}_{2} \mathrm{O}_{3}$ & 0,34 \\
\hline
\end{tabular}

Leucita) y el $\mathrm{Na}_{2} \mathrm{O}$. Este último, al igual que el $\mathrm{CaO}$ y el $\mathrm{MgO}$, son utilizados como fundentes. Los pequeños porcentajes de $\mathrm{SnO}$ y $\mathrm{Fe}_{2} \mathrm{O}_{3^{\prime}}$, son utilizados como opacificador y colorante, respectivamente. La distribución de tamaños de partícula de esta porcelana fue de 0.4 a $100 \mu \mathrm{m}$ con un tamaño promedio de $18 \mu \mathrm{m}$.

Acero inoxidable 304. A partir de una barra cilíndrica de acero inoxidable austenítico 304 de 1,27 cm de diámetro, se cortaron probetas de $0,5 \mathrm{~cm}$ de espesor. Las cuales fueron desbastadas con papeles abrasivos del número 80 al 2400 . Posteriormente, fueron pulidas a espejo con alúmina de 3 y $1 \mu \mathrm{m}, \mathrm{y}$ sometidas a un proceso de lavado por inmersión en una solución jabonosa a $95{ }^{\circ} \mathrm{C}$, por $5 \mathrm{~min}$. Posteriormente, fueron introducidas en agua a $65^{\circ} \mathrm{C}$, por $3 \mathrm{~min}$, lavadas en agua fría, y secadas con aire caliente.

\subsection{Preparación de las suspensiones.}

Los medios de dispersión empleados en la preparación de las suspensiones de porcelana fueron agua, etanol e isopropanol. La concentración de las suspensiones fue de 1 a $4 \%$ en peso. Las suspensiones fueron dispersadas ultrasónicamente durante $20 \mathrm{~min}$. De acuerdo al procedimiento descrito por Zhitomirsky y Gal-Or (10) las partículas más grandes de la suspensión fueron eliminadas por sedimentación durante 2 h. La aplicación de este procedimiento de sedimentación dio como resultado suspensiones de porcelana con un intervalo de tamaño de partículas de 1,5 a 2,3 mm. Para conocer algunas propiedades relacionadas con la movilidad electroforética de las partículas, se realizaron mediciones de potencial zeta $(\mathrm{z})$ a las diferentes suspensiones de porcelana en un Zetametro (Pem Kem, modelo 501) a una temperatura de $24^{\circ} \mathrm{C}$ utilizando aproximadamente $5 \mathrm{ml}$ de la suspensión original para cada medición.

\subsection{Equipo utilizado y condiciones empleadas para EPD.}

Para la realización de las pruebas de EPD se utilizó una celda electroforética de 0,5 1 de capacidad, una fuente de poder (BIO-RAD, modelo Power Pac 3000), un porta electrodo de trabajo de plástico donde se colocaron las probetas de acero inoxidable perfectamente aisladas y un contraelectrodo de platino, ambos electrodos con un área de trabajo de aproximadamente $0.95 \mathrm{~cm}^{2}$. La distancia entre electrodos fue alrededor de $1 \mathrm{~cm}$. En la figura 1, se muestra el esquema de la celda utilizada en la EPD de porcelana. Voltajes comprendidos entre 1 y $2000 \mathrm{~V}$ se aplicaron en todos los casos durante $3 \mathrm{~min}$. La 
cantidad de masa depositada fue calculada por diferencia entre el peso de la probeta utilizada y el peso conjunto de la probeta y el recubrimiento formado. Los valores de la cantidad de masa depositada fueron normalizados en todos los casos respecto al área de la probeta expuesta a la deposición y al tiempo de deposición seleccionado. La caracterización microestructural de los recubrimientos en verde obtenidos se llevó a cabo analizando su superficie en un microscopio electrónico de barrido (Philips, modelo XL30). Para determinar el efecto del contenido de sólidos sobre la cinética de deposición se utilizaron diferentes concentraciones $(2,3$ y $4 \%$ con cada medio de suspensión). Los espesores en verde fueron medidos por medio de un medidor de recubrimientos digital (Cole - Parmer, modelo U-59).

\section{RESULTADOS}

En la tabla 2 se muestran los resultados de las mediciones del potencial zeta. Las suspensiones en agua en comparación con las suspensiones preparadas con etanol e isopropanol, presentaron un potencial mucho mayor. También se puede observar, que los potenciales medidos en todas las suspensiones fueron negativos, lo que implicó, que las partículas de porcelana se cargaran negativamente en los tres medios de suspensión. Por lo tanto, el material fue depositado sobre el electrodo positivo en las pruebas de EPD.

Como se puede observar en la figura 2, cuando se utilizó agua como medio de suspensión, la velocidad de deposición electroforética de la porcelana fue mayor a voltajes considerablemente menores, con respecto al etanol y al isopropanol. En la tabla 3, se indica la cantidad máxima de masa depositada para cada medio de suspensión y los voltajes utilizados en su obtención. De igual forma, se muestra el rendimiento del voltaje con respecto a la cantidad de masa depositada para cada medio de suspensión. En esta tabla se puede apreciar que por cada voltio aplicado en una suspensión acuosa, se puede obtener un depósito 8,3 veces mayor que cuando se utiliza etanol, y 157 veces mayor que cuando se utiliza isopropanol.

Deposición en agua. En la figura 2, se puede observar que a medida que el voltaje aplicado se incrementa, la velocidad de deposición aumenta hasta llegar a un punto máximo. A partir de este máximo la velocidad de deposición disminuye. La apariencia de los depósitos en agua a diferente voltaje pueden observarse en las fotomicrografías de la figura 3. El depósito formado a $3 \mathrm{~V}$ no cubrió completamente la superficie del substrato (figura 3a). A $30 \mathrm{~V}$ se obtuvieron velocidades de deposición más elevadas, sin embargo el tamaño de partículas depositadas es mayor que al obtenido a $3 \mathrm{~V}$ (figura 3b). A voltajes superiores a $40 \mathrm{~V}$, se observó una disminución en el tamaño de las partículas depositadas (figura 3c) y en su velocidad de

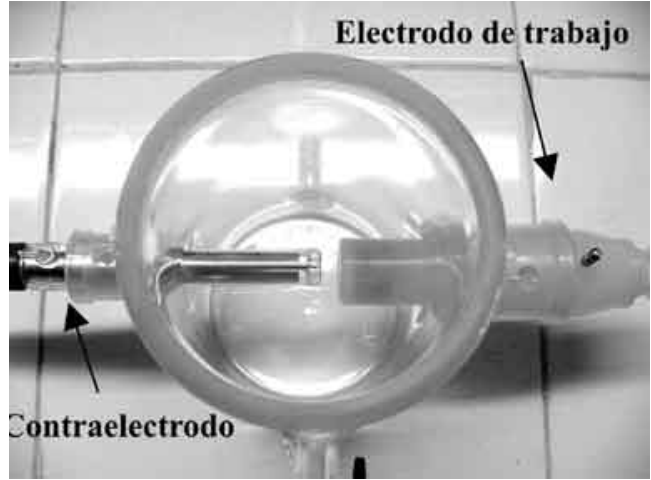

Figura 1 Esquema de la celda utilizada en la EPD de porcelana.

TABLA 2. Potencial ZeTA ( $\mathrm{z}$ ) DE LAS SUSPENSIONES UTILIZADAS

\begin{tabular}{|l|l|}
\hline \multicolumn{1}{|c|}{ Medio } & Potencial Zeta $(\mathrm{mV})$ \\
\hline Agua & $-34,16$ \\
\hline Etanol & $-5,2$ \\
\hline Isopropanol & $-1,6$ \\
\hline
\end{tabular}

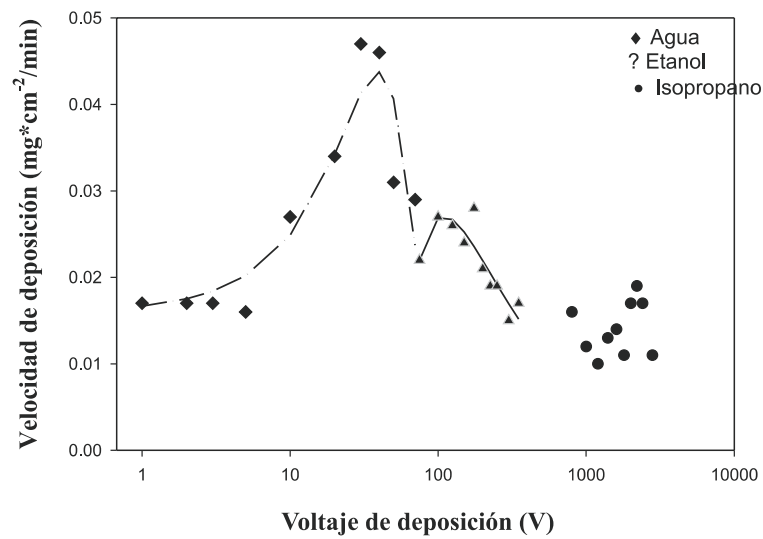

Figura 2 Cantidad de masa depositada en función del voltaje aplicado y del medio de suspensión de la porcelana en las pruebas de EPD

TABla 3. RENDIMIENTO DE LA CANTIDAD DE MASA DEPOSITADA PARA LOS DIFERENTES MEDIOS DE SUSPENSIÓN UTILIZADOS

\begin{tabular}{|ccccc|}
\hline $\begin{array}{c}\text { Medio } \\
\text { de } \\
\text { Suspensión }\end{array}$ & $\begin{array}{c}\text { Voltaje } \\
(\mathbf{V})\end{array}$ & $\begin{array}{c}\text { Velocidad } \\
\text { Máxima } \\
\left(\mathbf{m g}^{*} \mathbf{c m}^{-2} / \mathbf{m i n}\right)\end{array}$ & $\begin{array}{c}\text { Rendimiento } \\
\text { Vel. } \mathbf{m a ́ x} / \mathbf{V} \\
\left(\mathbf{m g}^{*} \mathbf{c m}^{-2} / \mathbf{m i n}^{*} \mathbf{V}\right)\end{array}$ & $\begin{array}{c}\text { Relación } \\
\mathbf{R}_{\max } / \mathbf{R}_{\mathbf{M}}\end{array}$ \\
\hline Agua & 30 & 0,047 & $1,5 \times 10^{-3}$ & 1 \\
\hline Etanol & 150 & 0,028 & $1,8 \times 10^{-4}$ & 8,3 \\
\hline Isopropanol & 2000 & 0,019 & $9,5 \times 10^{-6}$ & 157 \\
\hline
\end{tabular}

Nota: $R_{\max }$ es el rendimiento en (agua), y $\mathrm{R}_{\mathrm{M}}$ es el rendimiento en los otros medios
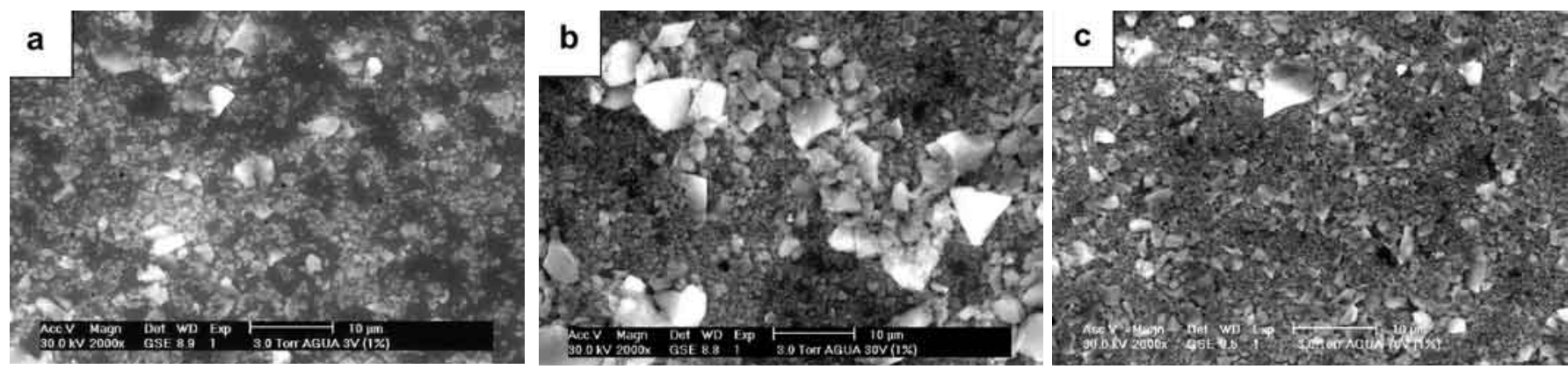

Figura 3 Fotomicrografías a 2000x de los depósitos de porcelana, utilizando como medio de suspensión agua, a diferentes voltajes: a) 3 V, b) 30 $\mathrm{V} \mathrm{y} \mathrm{c)} 70 \mathrm{~V}$. (Escala igual a $10 \mu \mathrm{m}$ ). 

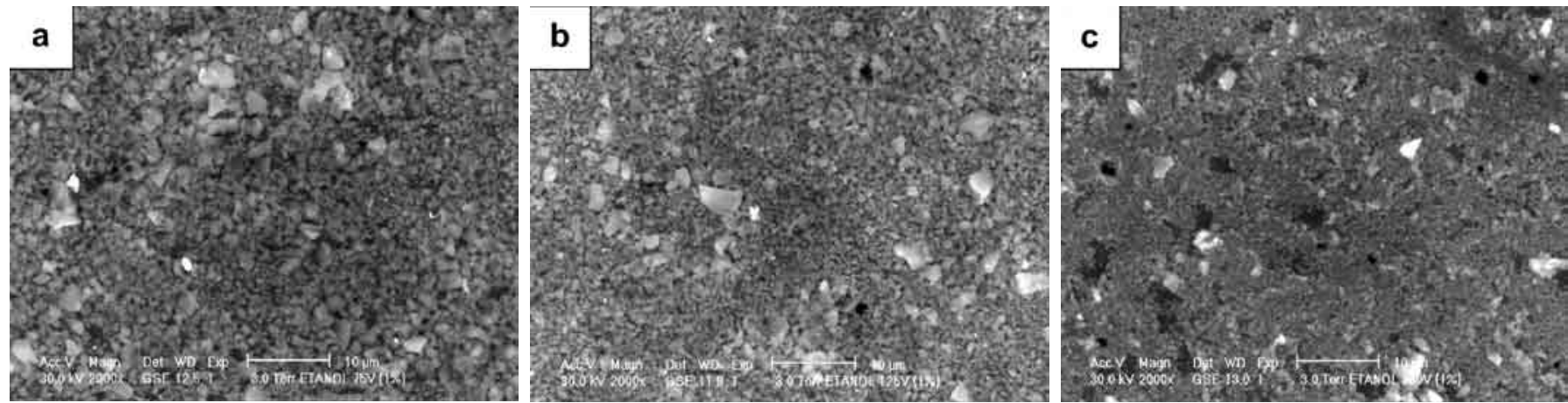

Figura 4 Fotomicrografías a 2000x de los depósitos en verde de porcelana, utilizando como medio de suspensión etanol, a diferentes voltajes: a) 75, b) 125 y c) $350 \mathrm{~V}$. (Escala igual a $10 \mu \mathrm{m}$ ).

deposición. El espesor obtenido a $40 \mathrm{~V}$ fue de $2.62 \mathrm{~mm}$.

De acuerdo a la ecuación de Henry (11), la velocidad de deposición se incrementa al aumentar el voltaje aplicado, ya que esto incrementa la movilidad electroforética de las partículas. De la misma forma se debería incrementar la presencia de partículas de mayor tamaño. Sin embargo, en la figura 3c, se puede observar que el depósito obtenido a $70 \mathrm{~V}$ en comparación con el obtenido a $30 \mathrm{~V}$ (figura $3 \mathrm{~b}$ ), contrario a lo esperado, presenta un depósito con partículas de menor tamaño. Por lo que podría inferirse, que la disminución en la velocidad de deposición podría ser ocasionada por la electrólisis del agua y problemas de corrosión en el electrodo de trabajo.

Deposición en etanol. Como se puede observar en la figura 2, el valor máximo de velocidad de deposición, y por lo tanto, el correspondiente a un mayor depósito se obtuvo entre 100 y 150 V. La aplicación de voltajes mayores redujo la cantidad de masa depositada. Este cambio en la cantidad de masa depositada, podría ser provocado por la degradación del electrodo de trabajo debido a la aplicación de voltajes muy elevados. Cuando se sobrepasa el potencial electroquímico de algún material, éste se descompone formando iones en la superficie del electrodo, los cuales afectan a la deposición de las partículas de porcelana disminuyendo la eficiencia del potencial aplicado, y por lo tanto, la cantidad de masa depositada $(3,4)$. Huellas de corrosión en la superficie del electrodo de trabajo fueron corroboradas visualmente en las pruebas a $300 \mathrm{~V}$.

En la figura 4, se muestra una serie de fotomicrografías de los depósitos en verde realizados en etanol a $75 \mathrm{~V}$ (figura 4a), $125 \mathrm{~V}$ (figura 4b) y $350 \mathrm{~V}$ (figura 4c). En la figura 4c, se pueden observar algunas zonas sin depósito, posiblemente generadas por burbujeo durante el ensayo, asociados a fenómenos electroquímicos en la interfase substrato-depósito. El espesor mayor del recubrimiento fue de 1,98 $\mu \mathrm{m}$ el cual fue obtenido a $125 \mathrm{~V}$.

Deposición en isopropanol. En la figura 2, se puede observar una gran dispersión en los valores obtenidos de la cantidad de masa depositada en función del voltaje aplicado durante las pruebas de EPD usando isopropanol como medio de suspensión. Esta tendencia puede estar asociada con el pobre nivel de estabilidad que presentó la porcelana en isopropanol ya que el valor del potencial zeta de esta suspensión fue de - 1.6. Por esta razón no fue posible determinar claramente una tendencia. Por otra parte, observaciones visuales mostraron marcas de corrosión en la superficie del electrodo de trabajo lo cual también pudo afectar la deposición de partículas.

Efecto de la concentración de sólidos sobre la cinética de deposición en los diferentes medios. En la figura 5, se presenta el efecto de la concentración de la porcelana en la suspensión contra la cantidad de masa depositada a voltaje constante, en diferentes medios de suspensión. En esta figura se puede observar que cuando se usa agua o etanol, la cantidad de masa depositada se incrementa casi en forma lineal conforme lo hace la concentración en la suspensión. En el caso de las suspensiones a base de isopropanol no se observó un efecto significativo en la cantidad de masa depositada, debido posiblemente a la falta de estabilidad de la suspensión y a

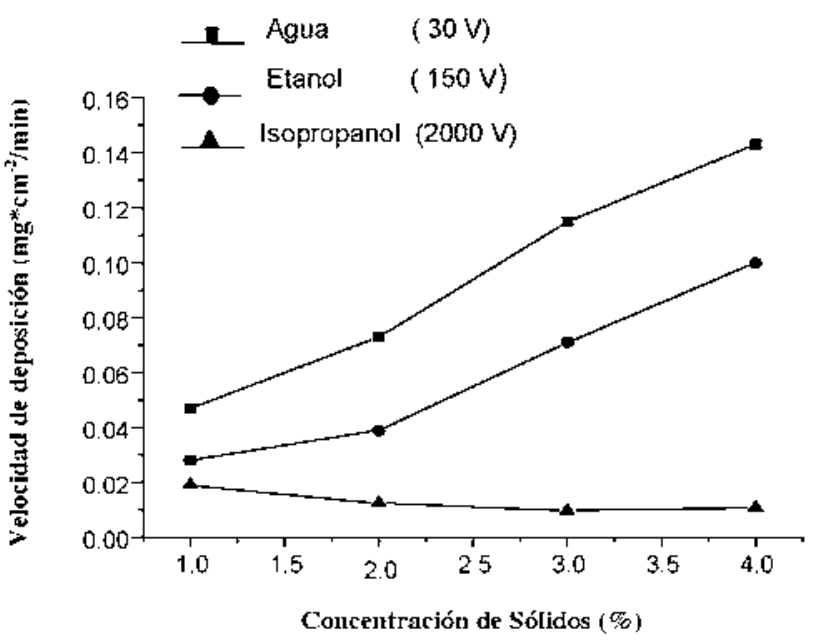

Figura 5 Efecto del medio de suspensión sobre la cantidad de masa depositada a diferentes concentraciones de porcelana. 

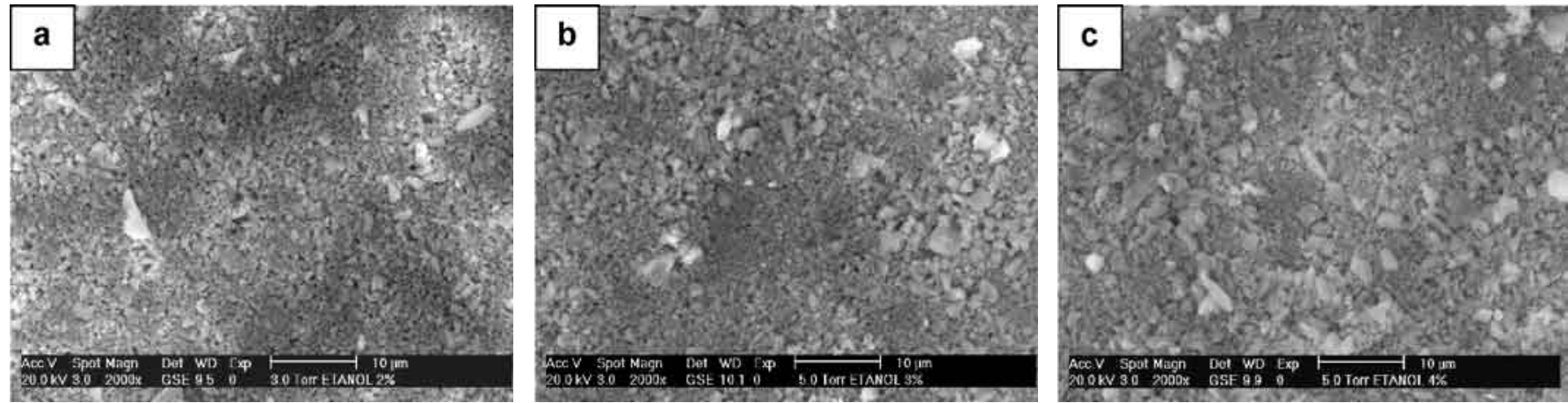

Figura 6 Fotomicrografías a 2000x de los depósitos en verde realizados en etanol a voltaje constante (150 V), utilizando diferentes concentraciones; a) $2 \%$, b) $3 \%$ y c) $4 \%$. (Escala igual a $10 \mu \mathrm{m}$ ).

problemas de corrosión en el electrodo de trabajo.

En la figura 6a-c, se muestra una serie de fotomicrografías tomadas a los depósitos realizados en etanol a voltaje constante $(150 \mathrm{~V})$, utilizando diferentes concentraciones de partículas de porcelana. En esta figura, se puede apreciar un ligero incremento en la cantidad de partículas de tamaño mayor. El cual pudo ser originado por la mayor concentración de aglomerados producida por los bajos niveles de repulsión entre partículas de acuerdo con el potencial zeta de la suspensión en etanol $(z=-5.2)$. El espesor máximo de recubrimiento obtenido en etanol fue de 2,12 $\mu \mathrm{m}$ cuando se aplicaron $150 \mathrm{~V}$ a una suspensión con 4\% de sólidos. Cuando se usó una suspensión de $4 \%$ de sólidos en agua, el espesor máximo fue de 2,75 $\mu \mathrm{m}$ cuando se le aplicaron $30 \mathrm{~V}$.

Acabado superficial. En la figura 7, se muestra una serie de fotomicrografías tomadas por microscopia estereográfica a depósitos en verde obtenidos con suspensiones base agua y etanol. Como se puede observar en la figura 7, el acabado superficial del depósito realizado con agua (figura 7a), presenta un aspecto muy irregular en comparación con los obtenidos en etanol a diferentes concentraciones de sólidos (figura 7b). Esta diferencia en el acabado superficial podría estar relacionada con los fenómenos que ocurren durante la deposición, como la formación de burbujas en el electrodo (electrólisis) para el caso del agua. Mientras que para el etanol, y a pesar de la relativa baja estabilidad de las suspensiones, se puede inferir que al tener una cinética de deposición lenta se originó un mejor acomodo de las partículas depositadas. El secado del depósito no influyó de manera importante en la formación de defectos como consecuencia de los espesores delgados obtenidos.

\section{CONCLUSIONES}

El agua fue el medio de suspensión donde se obtuvo la mayor velocidad de deposición electroforética de la porcelana dental. Sin embargo, es necesario tomar precauciones para evitar la descomposición electrolítica del agua y problemas relacionados con corrosión en el electrodo de trabajo.

Se pueden obtener depósitos de porcelana dental uniformes y con buen acabado superficial por EPD utilizando etanol como vehículo de suspensión. Para este tipo de suspensiones, el intervalo de voltaje óptimo fue de 70 a $150 \mathrm{~V}$ para un tiem-
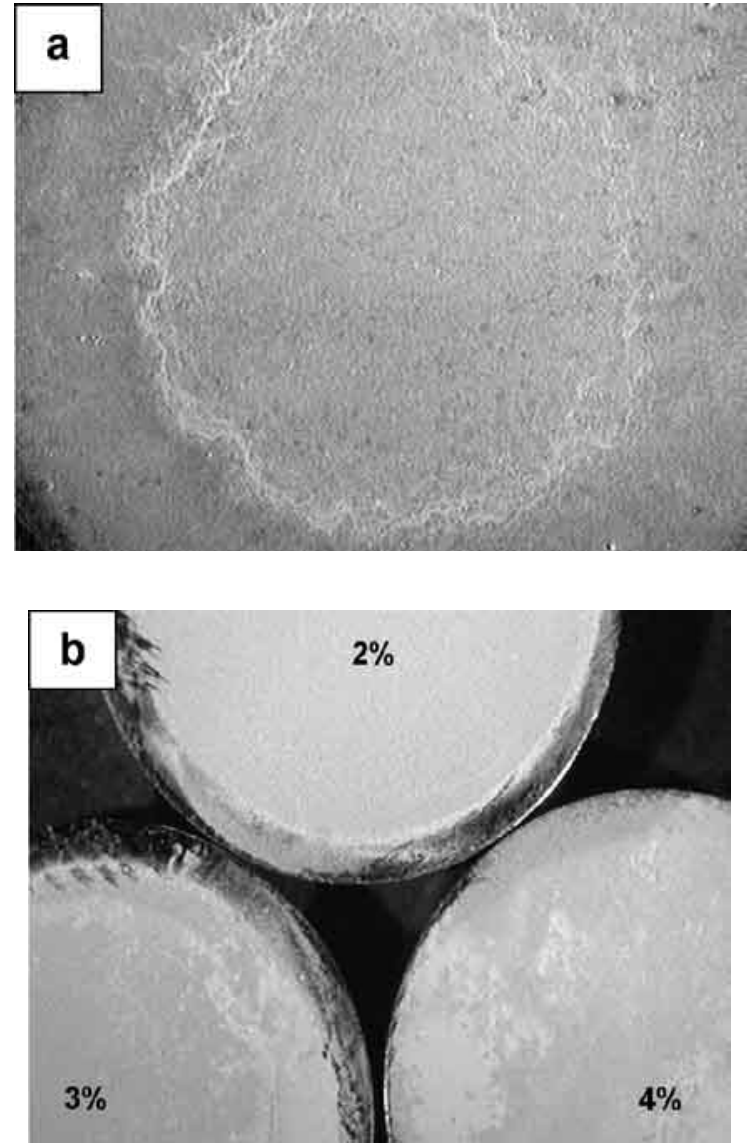

Figura 7 Fotomicrografías tomadas a los depósitos de porcelana en verde realizados con los diferentes medios de suspensión: (a) Agua, (b) etanol a 2, 3 y $4 \%$ de sólidos en etanol.

po de deposición de 3 min.

El uso de isopropanol generó depósitos heterogéneos debido a problemas relacionados con la baja estabilidad de las suspensiones y con reacciones electroquímicas en el electrodo 
de trabajo, derivadas de la aplicación de voltajes altos. BIBLIOGRAFÍA

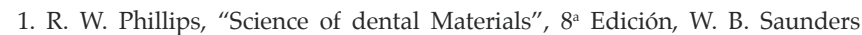
Company, pp. 502-531, 1982.

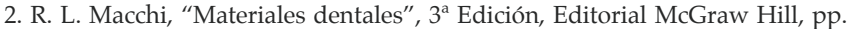
97-102, 257-311, 2000

3. B. Ferrari y R. Moreno, “Conformado de materiales cerámicos por electroforesis en medios acuosos", Bol. Soc. Esp. Cerám. Vidr, 37 (5) 368-381 (1998).

4. P. Sarkar y P. S. Nicholson, "Electrophoretic Deposition (EPD), mechanisms, kinetics, and aplicattion to ceramics", J. Amer. Ceram. Soc. 79 (8) 1987-2002 (1996).

5. B. Dibiás, "Coagulation and flocculation theory and applications", Vol. 47, Marcel Dekker, Inc., pp. 1-209 y 495-539, 1993.

6. B. Ferrari, J. C. Fariñas, y R. Moreno, “Determination and control of metallic impurities in alumina deposits obtained by aqueous electrophoretic deposition", J. Amer. Ceram. Soc. 84 (4) 733-739 (2001).

7. R. G. Horn, "Surface forces and their action in ceramic materials", J. Amer. Ceram. Soc. 73 (5) 1117-1135 (1990)

8. H. W. Hennicke, "Method and apparatus for coating dental crows and bridges", U. S. Patent No. 4,246,086, Jan. 20, 1981.

9. F. K. Gutersloh y W. Harsewinkel, "Method for electrophoretic enameling", United States Patent No. 4,466,871, Aug. 21, 1984.

10. I. Zhitomirsky y L. Gal-Or, "Electrophoretic deposition of hydroxyapatite" J.Mater. Sci.: Mater. Med, 8 213-219 (1997).

11. W. F. Smith, "Fundamentos de la ciencia e ingeniería de materiales", 3 Edición, Editorial McGraw Hill pp 495-540,1998.

Recibido: 26.09 .02

Aceptado: 25.11 .02

\section{UNTEQRR 2003 GONARESS}

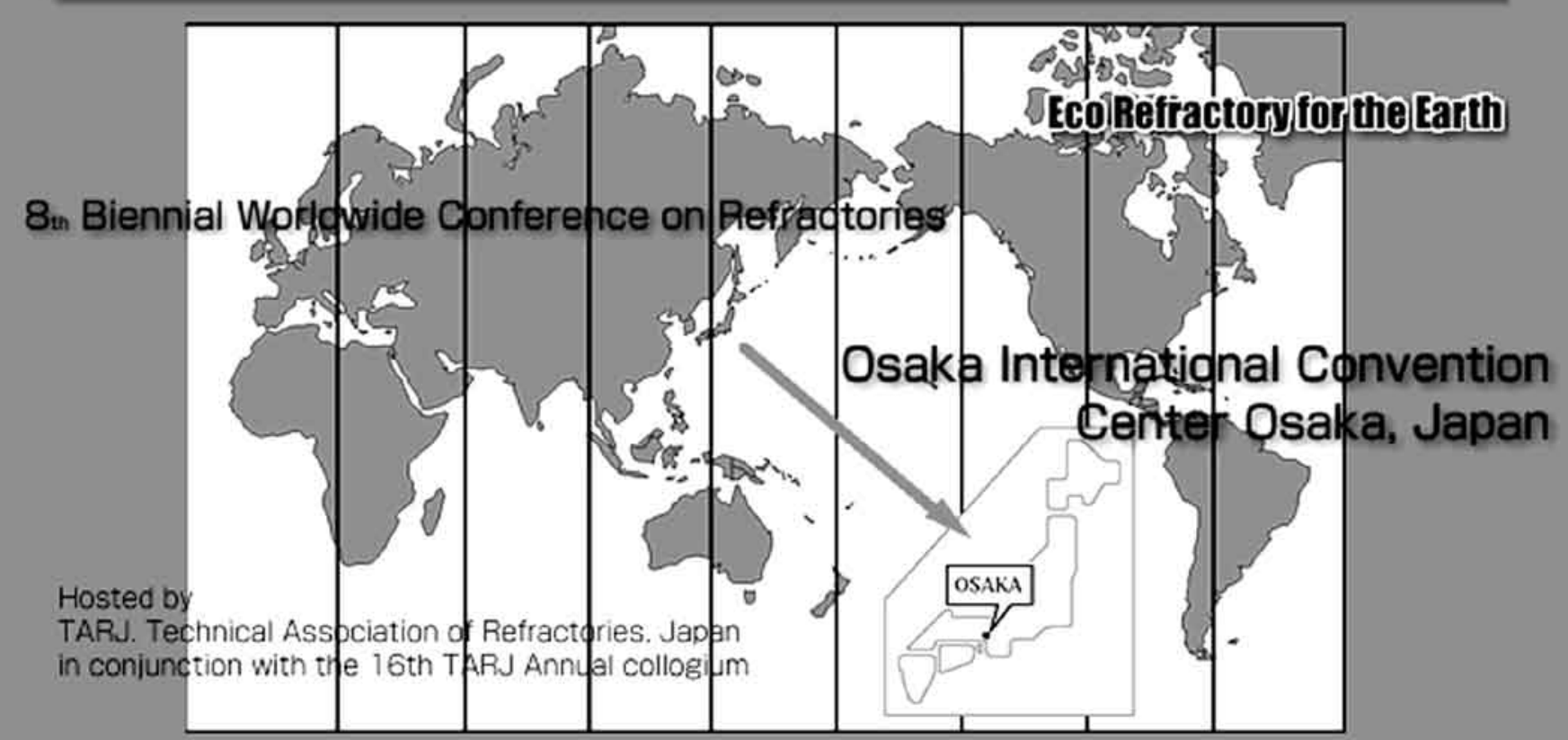

Otioher 19-22, 2003

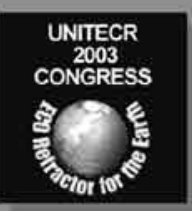

